\title{
Modeling the free convection in an open round cavity using a hybrid approach of Jaya optimization algorithm and neural network
}

\author{
Ehsan Akbari ${ }^{*}$, Ali-Mohammad Karami², Mehdi Ashjaee ${ }^{3}$ \\ ${ }^{1}$ Mechanical Engineering Department, University of Applied Science and Technology, Kermanshah 6714643759, Iran \\ ${ }^{2}$ Mechanical Engineering Department, Razi University, Kermanshah 6714967346, Iran \\ ${ }^{3}$ Mechanical Engineering Faculty, University of Tehran, Tehran 1417614418, Iran
}

Corresponding Author Email: Ehsan Akbari42@yahoo.com

https://doi.org/10.18280/ijht.360337

Received: 4 November 2017

Accepted: 28 August 2018

\section{Keywords:}

free convection, Jaya-based neural network, hybrid model, mach-zehnder interferometer, open round cavity

\begin{abstract}
The current study highlights the application of a hybrid model in which the Jaya optimization is employed to train the artificial neural network (ANN), to model the free convection in an open round cavity. As a matter of fact, the present research attempts to demonstrate the capability of the aforementioned hybrid network to model the free convection in the cavity against the decision parameters. The decision parameters are the Rayleigh number ( $\mathrm{Ra}$ ) and ratio of the nonconductor barrier distance from the bottom of the cavity to the cavity diameter $(\mathrm{H} / \mathrm{D})$. Then, the obtained hybrid model is applied to predict the average Nusselt number in the cavity. In the next step, the experimentally obtained data by using a Mach-Zehnder interferometer is used to train the hybrid model. The accuracy of the hybrid model is evaluated through the calculation of average testing and checking errors. According to the obtained results, there is an optimum ratio $(\mathrm{H} / \mathrm{D})$, in which the heat transfer is maximum. Also, this maximum value increases by increasing the Rayleigh number ( $\mathrm{Ra}$ ).
\end{abstract}

\section{INTRODUCTION}

The free convection heat transfer from cavities is frequently used in wide practical situations because of its easy access, cheapness and limited energy resources. Several studies have been focused on heat transfer in cavities.

When the flow that generated by temperature difference is sufficiently slow, the boundary layer will not formed in closed cavities [1].

Morrison and Tran [2] experimentally investigated the free convective flow structure generated by heat transfer in a vertical rectangular cavity. Through the study, significant deviations from the two commonly dimensional assumptions related to end wall conduction was observed. Flow structures taken by laser Doppler anemometry showed that in the case where the end walls are carefully kept insulated, horizontal velocity component parallel to the heat conducting plates, is small as compared with the vertical one.

Kublbee et al. [3] studied the forced convection in open ducts and cavities with different boundary conditions. It was found that, at small temperature differences with large geometric dimensions of the cavity, laminar flow can be observed even at high Rayleigh numbers. Graebel [4] investigated the influence of Prandtl number on the free convection in a rectangular cavity. In the study, temperature and velocity profiles and also the Nusselt number were obtained in terms of the length ratio and the Rayleigh and Prandtl numbers. One of the studies which have been devoted to square cavity in a channel was an investigation which was performed by Humphrey and Jacobs [5]. The results revealed that significant buoyant forces arise in the cavity where Richardson number was about 30 , while these were small in the channel where Richardson number was about 0.05 . The free convection in a square cavity at large Rayleigh numbers was numerically studied by Hyun and Lee [6]. In the investigation, the flow was initiated by instantaneously raising and lowering the temperatures on the opposing side walls. It was found that, the effect of the thermal boundary conditions on the horizontal surfaces appears to have a negligible influence on the time histories. Also, for the Prandtl number smaller than unity, an oscillatory approach to the steady states was detected only when the Rayleigh number was sufficiently high to render a strongly type of boundary layer flow. The effect of inclination on the free convection from the inside surface of elliptic tube was experimentally investigated by Elshazly et al. [7]. The results showed that, increasing the axial distance from the lower end of the elliptic tube results in temperature distributions. Furthermore, an optimum value for the local Nusselt number was observed near the upper end of the tube. A research on square and circular cavities was carried out by Ridouane and Campo [8] In the study, the effect of two active curved vertical sides and two inactive curved horizontal ones on the free convection performance was numerically studied. It was found that, the heat transfer enhancement provided by the circular cavity increases for low Rayleigh numbers, decreases for moderate Rayleigh numbers and practically vanishes for high Rayleigh numbers. Mohammed and Salman [9] performed an experimental investigation on the combined free and forced convection to attain the thermally developing flow in a uniformly heated vertical circular cylinder. In the study, an empirical correlation to attain the flow has been extracted to evaluate the average Nusselt number in terms of the Rayleigh and Reynolds numbers. In another study, Khelifi-Touhami et al. [10] studied the laminar free convection flow in a cylindrical cavity. The results showed that, by increasing the Rayleigh number, the 
buoyant uprising warm flow adjacent to the vertical wall increases. More studies on the free convection in the cavities can be found elsewhere [11-15].

The main focus of this study is employing the Jaya optimization algorithm to train the artificial neural network (ANN), and introduce a hybrid network called as the Jayatrained ANN. Then, the obtained hybrid system is applied to model the free convection heat transfer in an open round cavity. In fact, the obtained model is employed to predict the output parameter versus input ones including the Rayleigh number (Ra) and ratio of the nonconductor barrier distance from the bottom of the cavity to the cavity diameter (H/D). After that, the obtained hybrid model is applied to predict the average Nusselt number in the cavity. In the next step, the experimentally obtained data by using a Mach-Zehnder interferometer is utilized to train the hybrid model. Eventually, a network with the least error is recognized as the best predictor model.

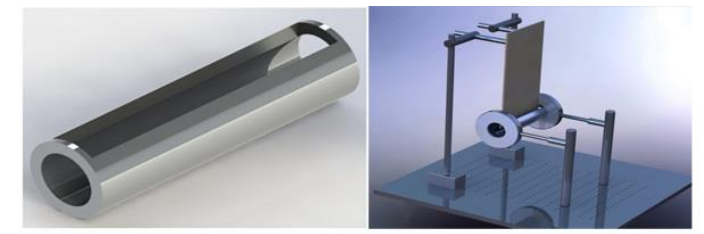

(a) (b)

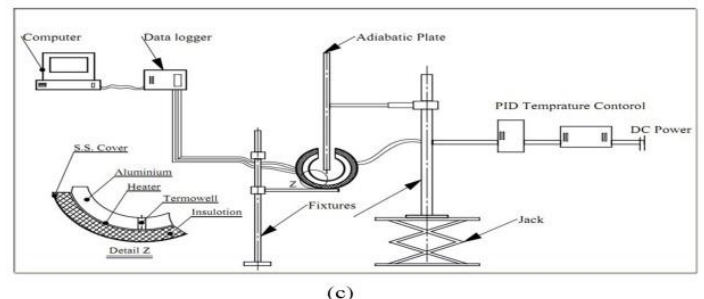

Figure 1. The schematic representation of the model geometry (a) Cylinder with top cavity configuration (b) Test section assembly (c) Experimental setup and control temperature system

Based on the authors' knowledge, there is not any research on the modeling of the free convection in open round cavities, using a hybrid model in which, the Jaya algorithm is used to train the artificial neural network. This non-contact method helps to have simultaneous undisturbed temperature fields with high accuracy. It is worth mentioning that, as indicated in the results section, for the values of the ratio (H/D) in excess of 3 , the experimental results are the same, therefore the H/D ratio of 3 is considered as infinity $(\infty)$. As a matter of fact, the $1 \infty$ ' represents the values in excess 3 for the H/D ratio. However, for the modeling process, the value of 3 is considered for the values in excess of 3 for this ratio. A schematic representation of the cavity with details is shown in Figure 1.

\section{THE MODELING APPROACH; PRINCIPLES}

In this section, the combination of the Jaya Optimization algorithm and artificial neural network (ANN) is considered. The Jaya algorithm (JA) is a new powerful population-based optimization technique introduced for the first time by Rao [16] to generate optimum solutions due to its computational capability when applied to the engineering optimization problems [17-23].
Unlike other population-based heuristic algorithms, the Jaya algorithm requires only few control parameters like population size $(m)$, which represents the number of candidate solutions and the number of generations $\left(G_{n}\right)$, which indicates the total iterations and the number of control parameters, which is often common among algorithms. The optimization process of the method is elicited on the basis of the idea that the solution determined for a special problem have to shift towards the optimum solution and evade the inferior solution. The main Jaya algorithm has only one phase according to the above-mentioned concept, making it a simple optimization method [24].

Moreover, unlike other population-based algorithms, the controlling of the algorithm parameters is not difficult. Also, controlling the parameters in each iteration is often easy and does not take much time. These two properties, shows the supremacy of the algorithm as compared to other algorithms. On the other hand, an artificial neuron network (ANN) [25] is regarded as a computational model on the basis of the structure and functions of biological neural networks. One of the most popular and applied neural network architectures is the feed forward multi-layer perceptron (MLP) network, which has been used for various engineering applications [26-29].

The modeling approach provides a framework to precisely describe the behavior of a system(s), including physical and nonphysical (informational, such as software) system(s), against the parameters affecting the system(s).

At first, the main problem is training the ANN, via an optimization algorithm. Several optimization algorithms for the heat transfer processes such as genetic algorithm (GA) [26], Heat transfer search (HTS) [27], particle swarm optimization (PSO) algorithm [28], the modified teaching-learning-based optimization algorithm [29], simulated annealing [30], generative design algorithm (GDA) [31], prey-predator algorithm [32], Global Best Algorithm [33] etc.

This process is performed using the Jaya optimization algorithm. For the first time in 2016, Trivedi et al. [34] employed the Jaya optimization algorithm to train the ANN, and introduced a hybrid network. The first important and fundamental step in training the ANN is adjusting the associated parameters of the ANN including the weights and biases.

In better words, the Jaya algorithm should attain a set of values for weights and biases which provides the highest level of prediction accuracy. Since the Jaya algorithm accepts the parameters in the form of a vector, the associated parameters of the ANN are defined for this algorithm as follows:

$\vec{V}=\{\vec{W}, \vec{\theta}\}=\left\{W_{1,1}, W_{1,2}, \ldots, W_{n, n}, \theta_{1}, \theta_{2}, \ldots, \theta_{j}\right\}$

where, $\mathrm{n}$ stands for the number of the input nodes, $W_{i j}$ is the connection weight from the $\mathrm{i}$-th node to the $\mathrm{j}$-th one, and $\theta_{j}$ represents the bias of the $j-t h$ hidden node.

After defining the parameters, the objective function needs to be defined for the Jaya algorithm. As stated above, the target in training an ANN is to attain the highest level of prediction accuracy for training, testing and checking data.

After all, the problem of training the ANN is briefly formulated with the parameter for the Jaya algorithm as follows:

Minimize : $F(\vec{V})=$ Average error 
The overall process of training the ANN via the Jaya algorithm is illustrated in Figure 2. As shown in this figure, the Jaya algorithm provides ANN with weights/biases and receives average error for all training data. The Jaya algorithm changes the weights/biases during an iterative process, to minimize the average error of all training data to the lowest possible value.

The conceptual model presented in Figure 3 also shows the manner of training the ANN through the Jaya algorithm.

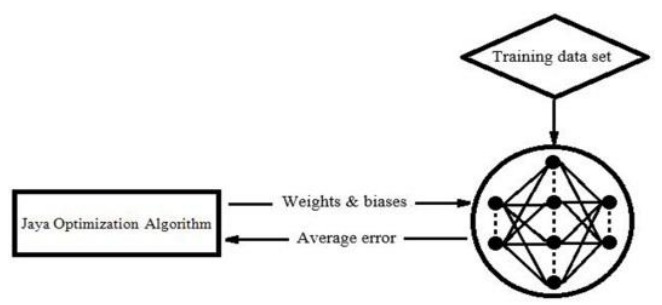

Figure 2. Simplified outline of the proposed hybrid model

\section{EXPERIMENTAL STUDY}

\subsection{Experimental setup}

\subsubsection{Interferometer}

The experimental study performed using the Mach-Zehnder interferometry (MZI) technique. The Mach-Zehnder interferometer constitutes of a light source, a pinhole, a micro lens, two doublets, two beam splitters and three mirrors [35]. The whole interferometer setup is schematically shown in Fig 3. Beam splitters of BS1 and BS2, along with plane mirrors of M1 and M2 are the main parts of the basic MZI. The laser beam expands after passing through spatial filter and doublet1. The expanded beam is split into two equal beams by the BS1. One beam passes through the test section and the other through the undisturbed field. These two beams, again, are recombined at BS2. If the four optical plates, M1, M2, BS1, and BS2 are parallel, then infinite fringe interferograms are formed. More information on the MZI is available elsewhere [35-37]. The applied light source was a $10 \mathrm{~mW}$ Helium - Neon laser with a $632.8 \mathrm{~nm}$ wavelength. All the interferograms are digitized by using a 'ARTCAM-320P' , 1/2" CCD $3.2 \mathrm{M}$ pixels camera. To take the interferograms, a camera is integrated with a PC. Fig 4 represents some of the interferograms which are recorded by the CCD camera.

\subsubsection{Experiment test section}

In this study a hollow aluminum cylinder with inner diameter of $45 \mathrm{~mm}$, thickness of $10 \mathrm{~mm}$ and length of $220 \mathrm{~mm}$ is used. Also cylinder is open on top with a length of $0.8 \mathrm{D}$, as it is shown in Figure 1-a. In order to achieve a very fine surface smoothness and minimize radiation heat transfer, the cylinder surface is polished.

At the ends of the cylinder, two adiabatic glass windows are used to prevent the axial air flow in or out of it. The Surface roughness of windows is 0.1 wave length of laser beam to prevent distortion in isothermal contour recording process by laser beam. Cylinder and windows are positioned in two Teflon leg caps with a thermal conductivity $0.05 \mathrm{~W} / \mathrm{mK}$ [38] to minimize the end heat transfer effects.

A bone fiber with $210 \mathrm{~mm}$ in width, $5 \mathrm{~mm}$ in thickness and $500 \mathrm{~mm}$ in height is employed to investigate the slot effect on heat transfer. Two different types of positioner are used to hold and adjust the cylinder and barrier in fixed place. Teflon leg caps are placed inside two-dimensional positioner. A onedimensional positioner is employed to adjust barrier in arbitrary distance from inner cylinder surface.

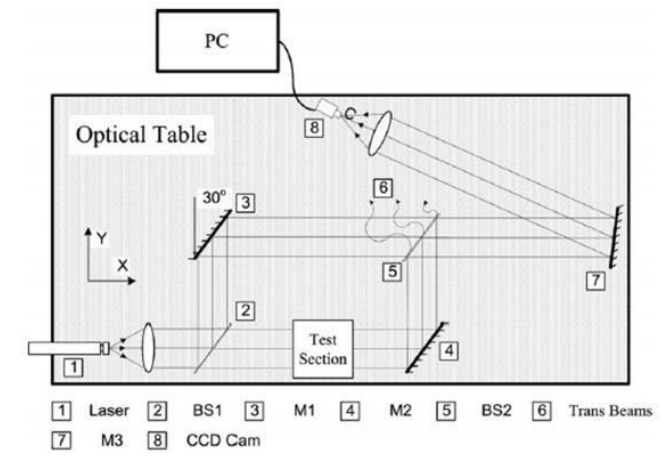

Figure 3. The schematic overview of the Mach-Zehnder interferometer

In order to achieve a constant temperature at the cylinder surface, three thin spiral rods of $\mathrm{Ni}-\mathrm{Cr}$ with electrical resistance of $3 \Omega$ is coated around cylinder as a heater. A mica paper is used as an electrical insulator between cylinder external surface and spiral rods. Heaters are isolated from ambient by a thermal insulator layer that restricts the heat transfer just to the cylinder. The power input to the heating elements is controlled by proportional-temperature-controller. These heaters are powered by $25 \mathrm{~V}-4 \mathrm{~A}$ DC power supply. By considering the above conditions, inner surface temperature of cylinder is remained uniform.

Four K-type thermocouples are inserted in the holes which are drilled in the cylinder wall. Two thermocouples are located in the stagnation line $(15 \mathrm{~mm}$ and $110 \mathrm{~mm}$ from the cylinder base) and two thermocouples are located in a line parallel to the stagnation line. The holes had a depth of $9.5 \mathrm{~mm}$. This means that the thermocouples had $0.5 \mathrm{~mm}$ distance from inner surface. The measured temperatures are the inner surface temperature because of high thermal conductivity of Aluminum. Maximum temperature difference among above thermocouples is less than $1^{\circ} \mathrm{C}$. So, constant temperature condition for inner surface of cylinder is held. All thermocouples are calibrated in an isothermal bath with accuracy $\pm 0.1^{\circ} \mathrm{C}$. All the temperatures are monitored continuously in a personal computer by a selector switch and a calibrated four channel data logger, TESTO 177 T4.

Ambient and reference temperatures are measured by two thermocouples. The reference temperature, $\mathrm{T}_{\text {ref, }}$ is the measured temperature of a fringe that is far enough from the cylinder surface, and its fringe shift ' $\varepsilon$ ' is assumed to be zero [39]. The pressure and relative humidity of laboratory are recorded during all experiments. The maximum uncertainties of pressure measurement for present study are $\pm 100 \mathrm{~Pa}$.

In order to ascertain accuracy of measurements, heater voltage and current are recorded in all the experiments. So, energy balance calculation is performed by estimating free convection heat transfer from the fringe patterns of the MachZehnder interferometer and measuring electrical power input to the heater. In order to adjust the position of the cylinder with the laser beam, an appropriate holding mechanism is used, which is shown in Figure 1-b. The cylinder is held by two holding rods that are connected to the Teflon end caps. The rods are connected to a special stand that could be adjusted to provide the cylinder vertical and horizontal movement. For 
accurate cylinder adjustment, a picture is taken and entered into an image processing program. This program helped authors to know that whether the cylinder is in the right position or not. The entire experimental setup is done on an anti-vibration table which has an air cushion. Figure 1-c shows a schematic overview of the experimental setup and control temperature system, in which the control temperature system is composed of power supply, power control, temperature control, and measuring instruments.

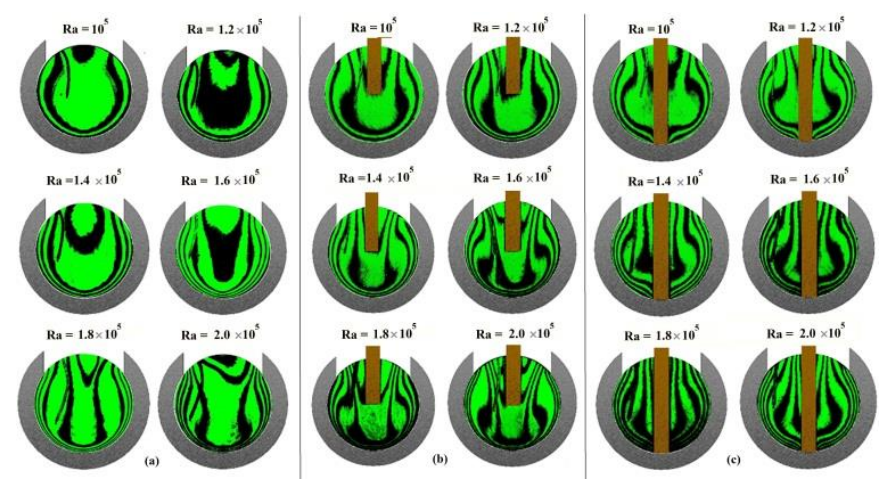

Figure 4. The interferograms of the cavity for (a) $H / D \rightarrow \infty$ (b) $\mathrm{H} / \mathrm{D}=0.5$ (c) $\mathrm{H} / \mathrm{D}=0$

\subsection{Data reduction procedure}

The main focus of the data reduction procedure is to determine local and average Nusselt numbers of inner cylinder surface at specified Rayleigh numbers. For each Rayleigh number, an interferogram is recorded. A MATLAB code is developed to calculate the temperature of the interference fringes (isotherms) as well as their distances from the cylinder surface at 11 circumferential locations $(0,10,20,30,40,50$, $60,70,80,90,100$ and 110 degrees from stagnation point), Fig 5 . The temperature distribution at each peripheral location in the inner surface thermal boundary layer of open cylinder is calculated by the method explained in detail by Teoch et al. [40]. Data reduction procedure for a sample interferogram is presented in Fig 5. A thermocouple is located inside the cylinder to measure reference temperature. The thermocouple is far enough from the surface in order to be outside the velocity and thermal boundary layers, which reduces flow and temperature fields' disturbance. The fringe, which is accordance thermocouple position, is considered as reference fringe with zero order number. Then by decreasing the radial distance towards the inner surface of the open cylinder, order number of the fringes increases and reaches the highest value at inner surface. Subsequently, temperature of each fringe is calculated based on the following relation [39]:

$T=\frac{3 P_{\infty} N_{r} l T_{r e f}}{3 l P_{\infty} N_{r}-2 \lambda R_{0} T_{r e f} \varepsilon}$,

where, $R_{0}=287 \mathrm{~J} / \mathrm{kg} . K$ represents the gas constant, $P_{\infty}$ shows the ambient pressure, $N_{r}=1.503 \times 10^{-4} \mathrm{~m}^{3} / \mathrm{kg}$ is the specific refractivity of air, $\lambda$ is the laser wave length, $l$ is the cylinder length along the laser beam, $T_{\text {ref }}$ stands for the reference temperature, and $\varepsilon$ represents the fringe shift. To evaluate temperature gradient at the surface, the variation of the fringe shift $\varepsilon$ versus radial distance is measured. Then the temperature gradient at the surface is calculated by differentiating of Eq. (3) with respect to the radial distance, as follows:

$\left.\frac{d T}{d r}\right|_{r}=\left.\left.\frac{d T}{d \varepsilon}\right|_{r} \cdot \frac{d \varepsilon}{d r}\right|_{r}$

$d T /\left.d \varepsilon\right|_{r}$ is calculated by differentiating Eq. (3) respect to as follows:

$\left.\frac{d T}{d \varepsilon}\right|_{r}=\frac{6 N_{r} l P_{\infty} \lambda R_{0} T_{r e f}^{2}}{\left(3 l P_{\infty} N_{r}-2 \lambda R_{0} T_{r e f} \varepsilon\right)_{r}^{2}}$

Therefore, the local convection heat transfer coefficient at each peripheral position is determined as follows:

$h_{L}=-\left.k \frac{d T}{d r}\right|_{r=D / 2}\left(\frac{1}{T_{w}-T_{\infty}}\right)$

where, $h_{L}$ represents the local heat transfer coefficient and $k$ stands for the air thermal conductivity at the cylinder surface temperature. Therefore,

$N u_{L}=\frac{h_{L} D}{k_{f}}=-\left.\frac{k D}{k_{f}\left(T_{w}-T_{\infty}\right)} \frac{d T}{d r}\right|_{r=D / 2}$

where, $N u_{L}$ indicates the local Nusselt number, $D$ is the diameter of open cylinder, and $k_{f}$ represents the air thermal conductivity at film temperature, which is defined as follows:

$T_{f}=\frac{T_{w}+T_{\infty}}{2}$

Finally, the average Nusselt number of the open cylinder for each case, $\overline{N u}$ is calculated as follows:

$\bar{h}=\frac{18}{11 \pi} \int_{0}^{\frac{11}{18 \pi}} h_{L} d \theta, \quad \overline{N u}=\frac{\bar{h} D}{k_{f}}$

where, $\theta$ is the periphery angle which is measured from the cylinder center.

\section{UNCERTAINTY ANALYSIS}

Experimental uncertainty analysis is a technique that analyzes a derived quantity, on the basis of the uncertainties in the experimentally measured quantities which are utilized in some form of mathematical relationship to calculate the derived quantity. In this study, the uncertainty analysis has been performed based on the method detailed elsewhere [3941]. The standard uncertainties in the fringe shift, gas constant, and the laser wave length are overlooked. Other uncertainties including the wall temperatures, ambient pressure and ambient temperature are calculated from the measuring devices precision and also the uncertainty related to the measuring devices of the cavity dimensions. The uncertainty of the difference in vertical distances of fringes from the surface and 
also thermal conductivity of air, are another significant parameters need to be considered. Whole error sources involved in the study are presented in Table 1. Considering the above mentioned uncertainties, the uncertainty in the measurement of local Nusselt numbers is calculated as $5.6 \pm$ $2.6 \%$.

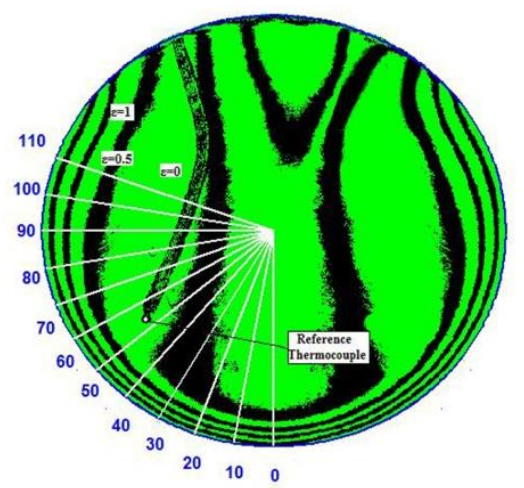

Figure 5. Data reduction procedure for a sample

Table 1. The error sources involved in the study

\begin{tabular}{cccc}
\hline Error Sources & Variables & $\begin{array}{c}\text { Bias } \\
\text { Errors }\end{array}$ & Units \\
\hline $\begin{array}{c}\text { Cavity dimensions } \\
\text { (distance of the } \\
\text { adiabatic plate from } \\
\text { the bottom of the } \\
\text { cylinder, length of the } \\
\text { cylinder, slot width, } \\
\text { diameter of the } \\
\text { cylinder) }\end{array}$ & $\mathrm{H}, 1, \mathrm{t}, \mathrm{D}$ & 0.08 & $\mathrm{~mm}$ \\
\hline Fringe distances & $\Delta \mathrm{r}$ & 0.02 & $\mathrm{~mm}$ \\
\hline Pressure & $\mathrm{P}$ & 100 & $\mathrm{~Pa}$ \\
\hline Temperature & $\mathrm{T}$ & 0.1 & ${ }^{\circ} \mathrm{C}$ \\
\hline $\begin{array}{c}\text { Thermal conductivity } \\
\text { of air }\end{array}$ & $\mathrm{k}$ & 0.0005 & $\begin{array}{c}\mathrm{W} / \\
\mathrm{m} .{ }^{\circ} \mathrm{K}\end{array}$ \\
\hline
\end{tabular}

\section{THE MODEL DEVELOPMENT}

For determining the average Nusselt numbers of inner cylinder surface $(\overline{N u})$, as the output parameters, an independent model was developed. The input parameters of this models was including the Rayleigh number $(\mathrm{Ra})$, ranging from $10^{5}$ to $2 \times 10^{5}$, and the ratio of the non-conductor barrier distance from the bottom of cavity to the cavity diameter (H/D), from 0 to 3. Indeed, a two-input/one-output model is employed in this investigation. The schematic overview of the suggested hybrid model has been presented in Figure 6.

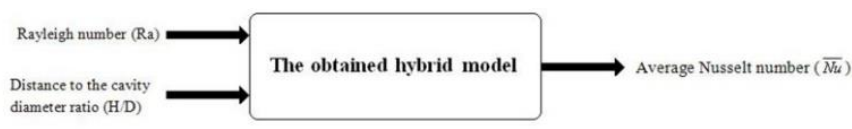

Figure 6. A simplified outline of the suggested hybrid model to predict the average Nusselt number $(\overline{\mathrm{Nu}})$

In order to develop the proposed model, 33 data are employed. The entire data are segregated into three sets: training, testing and checking. About $58 \%$ of the whole data (19 data) were employed for training, $27 \%$ for testing (9 data) and the left 15\% (5 data) were employed as checking data for evaluating the performance of proposed hybrid model. The training and testing sets were selected randomly.

The best architecture of the proposed hybrid model for predicting the average Nusselt number $(\overline{\mathrm{Nu}})$ is described in Table 2 .

Table 2. Optimal architecture and characteristic of the proposed hybrid model for predicting the average Nusselt

$$
\text { number }(\overline{N u})
$$

\begin{tabular}{|l|c|}
\hline Neural network type & MLP \\
\hline Number of hidden layers & 2 \\
\hline Inputs / Output & $2 / 1$ \\
\hline Number of neurons in the input layer & 2 \\
\hline Number of neurons in the first hidden layer & 3 \\
\hline Number of the weight parameters in the first hidden layer & 6 \\
\hline Number of the bias parameters in the first hidden layer & 3 \\
\hline Number of neurons in the second hidden layer & 4 \\
\hline Number of the weight parameters in the second hidden layer & 24 \\
\hline Number of the bias parameters in the second hidden layer & 4 \\
\hline Number of neurons in the output layer & 1 \\
\hline Number of the weight parameters in the output layer & 24 \\
\hline Number of the bias parameters in the output layer & 1 \\
\hline Maximum number of generations (total number of iterations, $\mathrm{G}_{\mathrm{n}}$ ) & 220 \\
\hline Population size (candidate solutions, m) & 60 \\
\hline$\eta_{1} \& \eta_{2}$ & between 0 and 1 \\
\hline
\end{tabular}

\section{RESULTS AND DISCUSSION}

A comparison between the predicted values using the hybrid model and experimentally obtained ones for training, testing and checking data, is presented in Figure 7. From this figure, it is evident that the predicted values using the proposed hybrid model are in good agreement with the experimental data with least error.

The prediction performance of the suggested hybrid model is evaluated through the sum-squared errors (SSE), the coefficient of determination percentage (R2\%) mean absolute percentage error (MAPE), root-mean square error (RMSE), and standard deviation (STD) values, which are calculated as follows:

$$
\begin{aligned}
& S S E=\sum_{i}^{n}\left(Y_{\text {Exp,i }}-Y_{\text {Pred, }}\right)^{2} \\
& R^{2} \%=\left(1-\frac{S S E}{\sum_{i=1}^{N} Y_{\text {Exp }, \mathrm{i}}^{2}}\right) \times 100
\end{aligned}
$$

MAPE $\%=\frac{1}{N} \sum_{i=1}^{N}\left|\frac{Y_{\text {Exp,i }}-Y_{\text {Pred, },}}{Y_{\text {Exp }, \mathrm{i}}}\right| \times 100$

$R M S E=\sqrt{\frac{\sum_{i}^{N}\left(Y_{\mathrm{Exp}, \mathrm{i}}-Y_{\mathrm{Pred}, \mathrm{i}}\right)^{2}}{N}}$

$S T D=\sqrt{\frac{\sum_{i}^{N}\left(Y_{\text {Exp }, \mathrm{i}}-Y_{\text {Pred, }}\right)^{2}}{N-1}}$ 
where, $\mathrm{N}$ is the number of data and ' $Y_{\text {Exp,i }}$ ' and ' $Y_{\text {Pred,i }}$ ' refer to experimentally obtained and predicted values, respectively.

The error information of the proposed hybrid model, in predicting the Nusselt number $(\overline{\mathrm{Nu}})$ is presented in Table 3 . According to this table, the MAPE\% and SSE for predicting the Nusselt number $(\overline{\mathrm{Nu}})$ based on the testing data set are $1.0023 \%$ and 0.0327 , respectively.

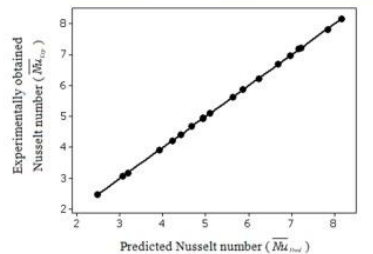

(a)

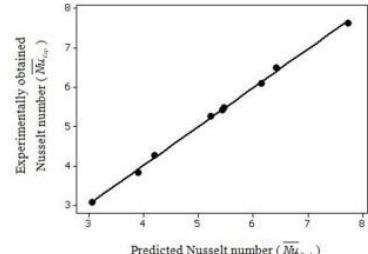

(b)

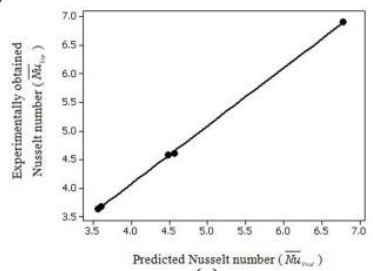

(c)

Figure 7. Comparisons between the experimentally obtained and the hybrid model predicted results for the (a) training (b) testing and (c) checking data sets

Also, Figure 8 shows the experimental results for the variation of local Nusselt number with angular distance from the bottom point of cylinder for all three positions of adiabatic plate in all Rayleigh numbers. This figure also clearly shows the increase of Nusselt number by increasing the Rayleigh number. According to this figure, in cases I and II, for all Rayleigh number, maximum Nusselt number occurs at bottom of cylinder and by going further distance, Nusselt number decreases continuously. This is expected because the boundary layer grows along this path (by increasing $\theta$ ). For all cases two symmetric vortexes have been created in two side of cavity. In the cases I and II, because of the vortex coincidence at the bottom of the cavity, no horizontal velocity component exists, so stagnation point is held at the lowest point of the cavity. Therefore, at this point, the thickness of the boundary layer is thin, and the heat transfer rate as well as the local Nusselt number are at their maximum values. Hence by increasing $\theta$, boundary layer grows and causes to decrease in the heat transfer rate. But in the case III, $\mathrm{H} / \mathrm{D}=0$, maximum Nusselt number doesn't occur at the bottom of cylinder and by moving further distance from adiabatic plate on the surface of the cavity, initially the Nusselt number increased and after a finite distance it starts to decrease. According to Figure 8, in this case, location of maximum Nusselt number is shifted slightly. Moreover, Figure 9 shows the variation of average Nusselt number $(\overline{N u})$ in terms of the H/D ratio for different Rayleigh numbers. By comparing the results presented in Figure 9, it can be observed that, the presence of the adiabatic plate increases the heat transfer rate. By increasing the H/D ratio from 0 (case III) to 0.5 (case II), the heat transfer increases ranging from $25 \%$ to $35 \%$. This manner can be due to the reason that, the adiabatic plate creates a velocity boundary layer around itself. By approaching the adiabatic plate to the bottom of the cavity (case II to case III), the velocity boundary layers of the adiabatic plate and surfaces overlap on each other and the cavity is separated into two halves. The heat transfer rate decreases due to overlapping of the boundary layers. Also, the bottom point of the cavity, which has the maximum heat transfer rate in cases I and II, is inactivated under the effect of adiabatic plate in case III. In earlier case, these effects are more visible in comparison to cases I and II. Hence although the Nusselt number in case III is greater than that in case I (without adiabatic plate), it is less than case II. By decreasing the H/D ratio from 3 (case I) to 0.5 (case II), the heat transfer increases from $25 \%$ to $35 \%$. In this regard, it can be deduced that the presence of adiabatic plate causes smaller vortexes. The thickness of the velocity boundary layer on the constant temperature surface decreases, whereas the velocity values inside the boundary layer increase. These effects cause to increase the heat transfer rate.

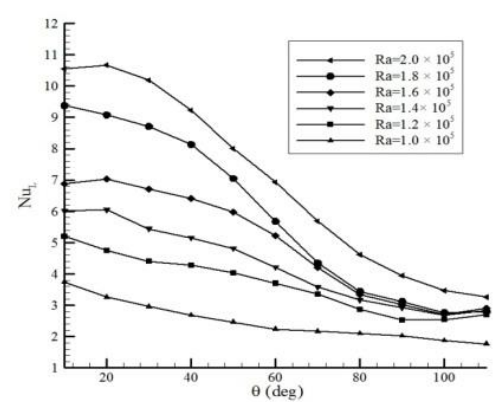

(a)

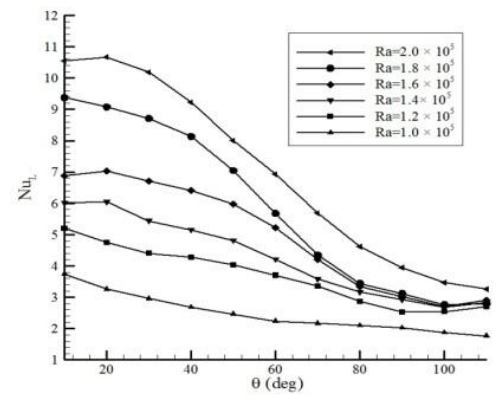

(b)

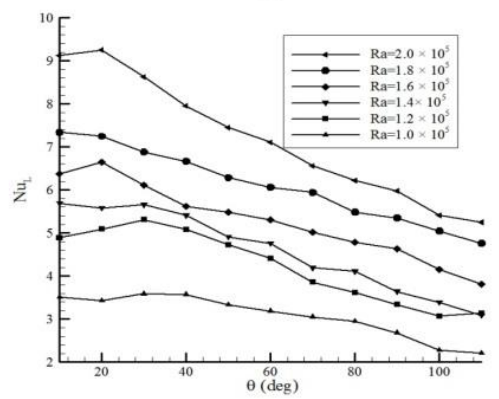

(c)

Figure 8. Experimental local Nusselt number versus Rayleigh numbers (a) case I (b) case II (c) case III

Table 3. The accuracy of the proposed hybrid model for predicting the average Nusselt number $(\overline{N u})$

\begin{tabular}{|c|c|c|c|c|c|c|c|c|}
\hline No & Output & \multicolumn{2}{|c|}{ Error bounds } & MAPE\% & SSE & $\mathrm{R}^{\mathbf{2 0}}$ & RMSE & STD \\
\hline 1 & $\begin{array}{c}\text { Nusselt } \\
\text { number } \\
(\overline{N u})\end{array}$ & Mean & Training & 0.1261 & 0.0024 & 99.9996 & 0.0113 & 0.0116 \\
\cline { 4 - 9 } & & Testing & 1.0023 & 0.0327 & 99.9877 & 0.0603 & 0.0640 \\
\cline { 5 - 9 } & & Checking & 1.7848 & 0.0376 & 99.9677 & 0.0867 & 0.0969 \\
\hline
\end{tabular}


Furthermore, it is worth mentioning that, the aforementioned hybrid models have been trained and tested using different numbers of hidden layers. The results are presented in Figure 10. As can be observed from these figures, for the training, testing and checking data, using two hidden layers, leads to better results in comparison with the one layer. But, increasing the number of hidden layers, does not have any sensible effect on the results. As can be observed, using two hidden layers, leads to $49 \%$ decrease in MAPE with respect to similar error by using only one hidden layer, for predicting the Nusselt number ( $\overline{\mathrm{Nu}}$ ), based on the testing data. The specifications of the compared hybrid models in which more hidden layers are applied, is presented in tables $4 \& 5$. As another result, it is important to be mentioned that increasing the number of hidden layers, decreases the convergence speed. In other words, increasing the number of hidden layers increases merely the time convergence of the model and computational complexity of the process.

As can be observed, using two hidden layers, leads to $49 \%$ decrease in MAPE with respect to similar error by using only one hidden layer, for predicting the Nusselt number $(\overline{\mathrm{Nu}})$, based on the testing data. The specifications of the compared hybrid models in which more hidden layers are applied, is presented in tables $4 \& 5$. As another result, it is important to be mentioned that increasing the number of hidden layers, decreases the convergence speed. In other words, increasing the number of hidden layers increases merely the time convergence of the model and computational complexity of the process.

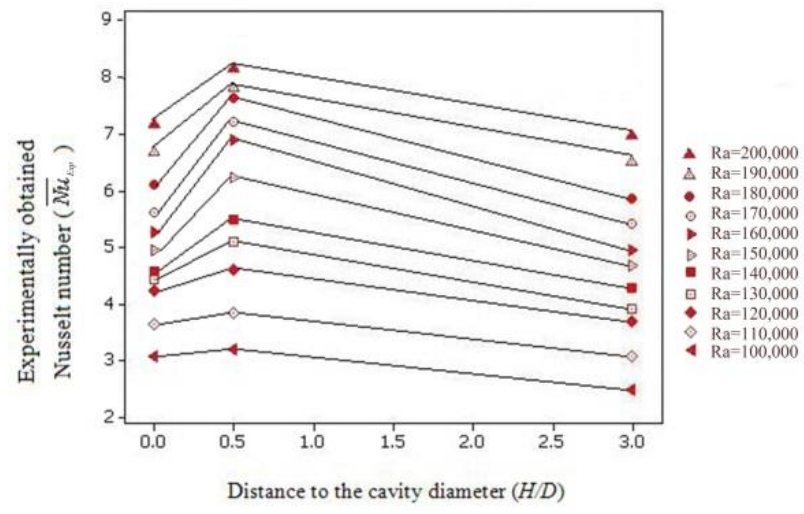

Figure 9. The average Nusselt number $(\overline{N u})$ versus the H/D ratio for different Rayleigh numbers $(\mathrm{Ra})$

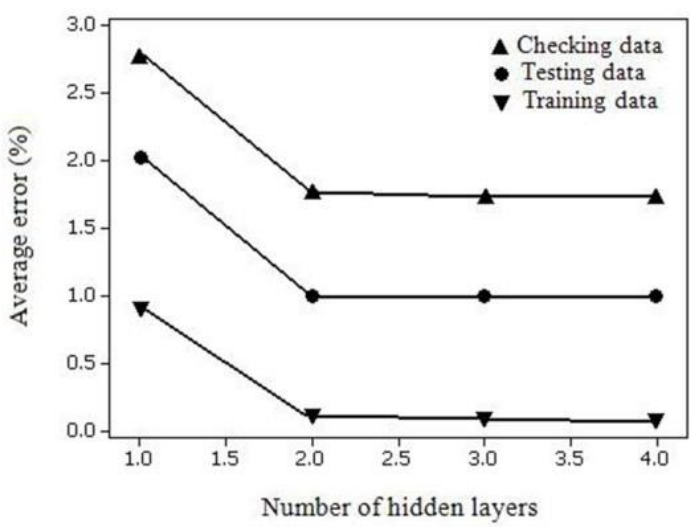

Figure 10. The MAPE\% of the hybrid model against the number of hidden layers for predicting the Nusselt number
Table 4. Optimal architecture and specifications of the first compared hybrid model for predicting the average Nusselt number $(\overline{N u})$

\begin{tabular}{|l|c|}
\hline Neural network type & MLP \\
\hline Number of hidden layers & 2 \\
\hline Inputs / Output & $2 / 1$ \\
\hline Number of neurons in the input layer & 2 \\
\hline Number of neurons in the first hidden layer & 2 \\
\hline Number of the weight parameters in the first hidden layer & 4 \\
\hline Number of the bias parameters in the first hidden layer & 2 \\
\hline Number of neurons in the second hidden layer & 2 \\
\hline Number of the weight parameters in the second hidden layer & 8 \\
\hline Number of the bias parameters in the second hidden layer & 2 \\
\hline Number of neurons in the third hidden layer & 3 \\
\hline Number of the weight parameters in the third hidden layer & 24 \\
\hline Number of the bias parameters in the third hidden layer & 3 \\
\hline Number of neurons in the fourth hidden layer & 3 \\
\hline Number of the weight parameters in the fourth hidden layer & 72 \\
\hline Number of the bias parameters in the fourth hidden layer & 3 \\
\hline Number of neurons in the output layer & 1 \\
\hline Number of the weight parameters in the output layer & 72 \\
\hline Number of the bias parameters in the output layer & 1 \\
\hline Maximum number of generations (total number of iterations, $\mathrm{G}_{\mathrm{n}}$ ) & 280 \\
\hline Population size (candidate solutions, $\mathrm{m}$ ) & 80 \\
\hline$\eta_{1} \& \eta_{2}$ & between 0 and 1 \\
\hline
\end{tabular}

Table 5. Optimal architecture and specifications of the second compared hybrid model for predicting the average Nusselt number $(\overline{N u})$

\begin{tabular}{|l|c|}
\hline Neural network type & MLP \\
\hline Number of hidden layers & 2 \\
\hline Inputs / Output & $2 / 1$ \\
\hline Number of neurons in the input layer & 2 \\
\hline Number of neurons in the first hidden layer & 2 \\
\hline Number of the weight parameters in the first hidden layer & 4 \\
\hline Number of the bias parameters in the first hidden layer & 2 \\
\hline Number of neurons in the second hidden layer & 2 \\
\hline Number of the weight parameters in the second hiddenlayer & 8 \\
\hline Number of the bias parameters in the second hidden layer & 2 \\
\hline Number of neurons in the third hidden layer & 3 \\
\hline Number of the weight parameters in the third hidden layer & 24 \\
\hline Number of the bias parameters in the third hidden layer & 3 \\
\hline Number of neurons in the fourth hidden layer & 3 \\
\hline Number of the weight parameters in the fourth hidden layer & 72 \\
\hline Number of the bias parameters in the fourth hidden layer & 3 \\
\hline Number of neurons in the output layer & 1 \\
\hline Number of the weight parameters in the output layer & 72 \\
\hline Number of the bias parameters in the output layer & 1 \\
\hline Maximum number of generations (total number of iterations, $\mathrm{G}_{\mathrm{n}}$ ) & 280 \\
\hline Population size (candidate solutions, m) & 80 \\
\hline$\eta_{1} \& \eta_{2}$ & between 0 and 1 \\
\hline
\end{tabular}

\section{CONCLUSIONS}

In this paper, a hybrid network in which the Jaya optimization algorithm is employed to train the artificial neural network was introduced to predict the free convection in an open round cavity against the inputs parameters. The input parameters were the Rayleigh number $(\mathrm{Ra})$ and ratio of the nonconductor barrier distance from the bottom of the cavity to the cavity diameter (H/D). The main idea of the paper was to develop a hybrid approach for modeling the output parameter as a function of input ones. As a matter of fact, a two-input/one-output network was applied in the investigation. The aforementioned network was developed based on the experimentally obtained data. It was concluded that, the hybrid network can precisely predict the results due to its low error rate. Moreover, the results demonstrated that, there is an optimum ratio (H/D), in which the heat transfer is maximum. Furthermore, it was observed that the best predictor model 
which utilizes two hidden layers, gives better results than those use more than two hidden layers.

\section{REFERENCES}

[1] Ostroumov GA. (1965). Free convection in closed cavities: A review of work carried out at PERM, USSR. International Journal of Heat and Mass Transfer 8(2): 259-268. https://doi.org/10.1016/0017-9310(65)90113-4

[2] Morrison GL, Tran VQ. (1978). Laminar flow structure in vertical free convective cavities. International Journal of Heat and Mass Transfer 21(2): 203-213. https://doi.org/10.1016/0017-9310(78)90224-7

[3] Kublbec K, Merker GP, Strawi J. (1980). Advance numerical computation of two-dimensional time dependent free convection in cavities. International Journal of Heat and Mass Transfer 23(2): 203-217. https://doi.org/10.1016/0017-9310(80)90197-0

[4] Graebel WP. (1981). The influence of Prandtl number on free convection in a rectangular cavity. International Journal of Heat and Mass Transfer 24(1): 125-131. https://doi.org/10.1016/0017-9310(81)90100-9

[5] Humphrey JAC, Jacobs EW. (1981). Free-forced laminar flow convective heat transfer from a square cavity in a channel with variable inclination. Int. J. of Heat and Mass Transfer 24: 1589-1597. https://doi.org/10.1016/00179310(81)90067-3

[6] Hyun JM, Lee JW. (1989). Numerical solutions for transient natural convection in a square cavity with different sidewall temperatures. International Journal of Heat and Fluid Flow 10(2): 146-151. https://doi.org/10.1016/0142-727X(89)90009-X

[7] Elshazly K, Moawed M, Ibrahim E, Emara M. (2005). Heat transfer by free convection from the inside surface of the vertical and inclined elliptic tube. Energy Conversion and Management 46(9-10): 1443-1463. https://doi.org/10.1016/j.enconman.2004.07.003

[8] Ridouane EH, Campo A. (2006). Free convection performance of circular cavities having two active curved vertical sides and two inactive curved horizontal sides. Applied Thermal Engineering 26(17-18): 2409-2416. https://doi.org/10.1016/j.applthermaleng.2006.02.019

[9] Mohammed HA, Salman YK. (2007). Combined natural and forced convection heat transfer for assisting thermally developing flow in a uniformly heated vertical circular cylinder. International Communications in Heat and Mass Transfer 34(4): 474-491. https://doi.org/10.1016/j.icheatmasstransfer.2007.01.001

[10] Khelifi-Touhami MS, Benbrik A, Lemonnier D, Blay D. (2010). Laminar natural convection flow in a cylindrical cavity application to the storage of LNG. Journal of Petroleum Science and Engineering 71(3-4): 126-132. https://doi.org/10.1016/j.petrol.2009.12.011

[11] Sun Q, Pop I. (2011). Free convection in a triangle cavity filled with a porous medium saturated with nanofluids with flush mounted heater on the wall. International Journal of Thermal Sciences 50(11): 2141-2153. https://doi.org/10.1016/j.ijthermalsci.2011.06.005

[12] Akbari E, Karami A, Ashjaee M. (2018). A comparison between radial basis function (RBF) and adaptive neurofuzzy inference system (ANFIS) to model the free convection in an open round cavity. Heat Transfer Asian Research. https://doi.org/10.1002/htj.21355
[13] Lipnicki Z, Weigand B. (2012). An experimental and theoretical study of solidification in a free-convection flow inside a vertical annular enclosure. International Journal of Heat and Mass Transfer 55(4): 655-664. https://doi.org/10.1016/j.ijheatmasstransfer.2011.10.044

[14] Karami A, Veysi F, Mohebbi S, Ghashghaei D. (2014). Optimization of laminar free convection in a horizontal cavity consisting of flow diverters using ICA. Arab J Sci Eng. 39(3): 2295-2306. https://doi.org/10.1007/s13369013-0741-8

[15] Triveni MK, Panua R. (2017). Numerical analysis of natural convection in a triangular cavity with different configurations of hot wall. International Journal of Heat and Technology 35(1): 11-18. https://doi.org/10.18280/ijht.350102

[16] Minai AA, Williams RD. (1990). Back-propagation heuristics: A study of the extended delta-bar-delta algorithm. Joint Conference on Neural Networks, Atlantic City, pp. I595-I600.

[17] Amiri A, Karami A, Yousefi T, Zanjani M. (2012). Artificial neural network to predict the natural convection from vertical and inclined arrays of horizontal cylinders. Polish Journal of Chemical Technology 14(4): 46-52. https://doi.org/10.2478/v10026-012-0101-6

[18] Karami A, Rezaei E, Rahimi M, Zanjani M. (2012). Artificial neural modeling of the heat transfer in an air cooled heat exchanger equipped with butterfly inserts. International Energy Journal 13: 21-28.

[19] Khorsandi M, Feghhi SAH, Salehizadeh A, Roshani GH. (2013). Developing a gamma ray fluid densitometer in petroleum products monitoring applications using Artificial Neural Network. Radiation Measurements 59: 183-187. https://doi.org/10.1016/j.radmeas.2013.06.007

[20] Haykin S. (1994). Neural networks: A comprehensive foundation. Chapter 3, New York: Macmillan College Publishing Company, 191-199.

[21] Venkata Rao R. (2016). Jaya: A simple and new optimization algorithm for solving constrained and unconstrained optimization problems. International Journal of Industrial Engineering Computations 7(1): 1934. https://doi.org/10.5267/j.ijiec.2015.8.004

[22] Warid W, Hizam H, Mariun N, Abdul-Wahab NI. (2016). Optimal power flow using the Jaya algorithm. Energies 9(9): 678. https://doi.org/10.3390/en9090678

[23] Zhang Y, Yang X, Cattani C, Rao RV, Wang S, Phillips P. (2016). Tea category identification using a novel fractional fourier entropy and Jaya algorithm. Entropy 18(3): 77. https://doi.org/10.3390/e18030077

[24] Rao RV, Rai DP. (2017). Optimization of submerged arc welding process parameters using quasi-oppositional based Jaya algorithm. Journal of Mechanical Science and Technology 31(5): 2513-2522. https://doi.org/10.1007/s12206-017-0449-x

[25] Rao RV, Rai DP, Ramkumar J, Balic J. (2016). A new multiobjective Jaya algorithm for optimization of modern machining processes. Advances in Production Engineering and Management 11(4): 271-286. https://doi.org/10.14743/apem2016.4.226

[26] Ghanadi Arab MM, Hajabdollahi M, Hajabdollahi H. (2013). Multi-objective optimization of a fin with twodimensional heat transfer using NSGA-II and ANN. Journal of Applied Mechanical Engineering 2(2) 117 124. https://doi.org/10.4172/2168-9873.1000117 
[27] Patel VK, Savsani VJ. (2015). A novel optimization algorithm. Information Sciences, Heat Transfer Search (HTS) 324: 217-246. https://doi.org/10.1016/j.ins.2015.06.044

[28] Merma AG, Olivera CAC, Torem ML, Santos BF (2018). Comparison study of hematite bioflotation by $\mathrm{r}$. erythropolis and its biosurfactant: experiments and neural network modeling. Chemical Engineering Transactions 65: 439-444 https://doi.org/10.3303/CET1865074

[29] Fidalgo JLG, Valim IC, Rego ASC, Nachez JL, Vilani C, Martins ARFA, Santos BF. (2018). Neural network modeling for prediction of oxidized lignin content by delignification of sugarcane bagasse through hydrogen peroxide with RAMAN spectroscopy data. Chemical Engineering Transactions 65: 517-522. https://doi.org/10.3303/CET1865087

[30] Wu X, Kaifeng W, Xiaobin J, Gaohong H. (2016). Optimization of a shell and tube heat exchanger based on a genetic simulated annealing algorithm. Journal of Tsinghua University(Science and Technology) 56(7): 728-734. https://doi.org/10.16511/j.cnki.qhdxxb.2016.24.021

[31] Lohan DJ, Dede EM, Allison JT. (2016). Topology optimization for heat conduction using generative design algorithms. Structural and Multidisciplinary Optimization 55(3): 1063-1077. https://doi.org/10.1007/s00158-016-1563-6

[32] Hamadneh N, Khan W, Tilahun S. (2018). Optimization of microchannel heat sinks using prey-predator algorithm and artificial neural networks. Machines 6(2): 26-44. https://doi.org/10.3390/machines6020026

[33] Turgut OE. (2017). Multi-objective thermal design optimization of a shell and tube condenser through global best algorithm. Journal of Science and Engineering 19(56):

$644-665$ https://doi.org/10.21205/deufmd.2017195659

[34] Trivedi IN, Jangir P, Bhoye M, Jangir N, Parmar SA, Kumar A. (2016). Training multi-layer perceptrons in neural network using Jaya algorithm. IEEE (CSNT2016). https://doi.org/10.13140/RG.2.1.1265.3208

[35] Hauf W, Grigull U. (1970). Optical methods in heat transfer. Advances in Heat Transfer, Academic Press, New York, pp. 133-366.

[36] Eckert ERG, Goldstein RJ. (1972). Measurements in Heat Transfer, 2nd ed. McGraw-Hill, New York, pp. 241-293.

[37] Flack RD. (1978). Mach-Zehnder interferometer errors resulting from test section misalignment. Applied Optics 17(7): 985-987. https://doi.org/10.1364/AO.17.000985

[38] Bever MB. (1986). Encyclopedia of Materials Science and Engineering, Pergamon Press, Oxford, 4948-4952.

[39] Naylor D, Duarte N. (1999). Direct temperature gradient measurement using interferometry. Experimental Heat Transfer 12(4): 279-294. https://doi.org/10.1080/089161599269609

[40] Teoch PL, Shirinzadeh B, Foong CW, Alici G. (2002). The measurement uncertainties in the laser Interferometry based sensing and tracking technique. Measurement 32(2): 135-150. https://doi.org/10.1016/S0263-2241(02)00006-4

[41] Kline SJ, McClintock FA. (1953). Describing experimental uncertainties in single sample experiments. Mechanical Engineering 75(1): 3-8.

\section{NOMENCLATURE}

$\begin{array}{ll}\text { ANN } & \text { Artificial Neural Network } \\ \mathrm{D} & \text { Diameter of the cylinders }(\mathrm{m}) \\ \mathrm{g} & \text { Gravitational acceleration }(\mathrm{m} / \mathrm{s} 2) \\ \mathrm{h} & \text { Heat transfer coefficient }\left(\mathrm{W} / \mathrm{m} 2 .{ }^{\circ} \mathrm{K}\right) \\ \mathrm{H} & \text { Distance of adiabatic plate from the bottom of } \\ & \text { cylinder }(\mathrm{m}) \\ \mathrm{k} & \text { Thermal conductivity of air }\left(\mathrm{W} / \mathrm{m} .{ }^{\circ} \mathrm{K}\right) \\ 1 & \text { Cylinder length }(\mathrm{m}) \\ \mathrm{MAPE} & \text { Mean absolute percentage error, } \% \\ \mathrm{Nr} & \text { Specific refractivity of air }(\mathrm{m} 3 / \mathrm{kg}) \\ \mathrm{Nu} & \text { Nusselt number } \\ \mathrm{P} & \text { Pressure }(\text { Pa) } \\ \mathrm{r} & \text { Radius of the cylinders }(\mathrm{m}) \\ \mathrm{R} 2 & \text { Correlation coefficients } \\ \text { R0 } & \text { Gas constant }(\mathrm{J} / \mathrm{Kg} . \mathrm{K}) \\ \text { Ra } & \text { Rayleigh number based on the cylinder diameter } \\ & (\mathrm{g} \beta(\text { Tw-T } \infty) \mathrm{D} 3 / \mathrm{v} \alpha) \\ \mathrm{RMSE} & \text { Root-Mean Square Error } \\ \text { STD } & \text { Standard Deviation } \\ \text { SSE } & \text { Sum-Squared Errors } \\ \mathrm{t} & \text { Slot width }(\mathrm{m}) \\ \mathrm{T} & \text { Temperature }\left({ }^{\circ} \mathrm{K}\right)\end{array}$

\section{Greek symbols}

$\beta$

$\mu$

$\rho$

$\varepsilon$

$\theta$

$\lambda$

Subscripts
$\infty$
f
L
ref
W

Thermal diffusivity of air $(\mathrm{m} 2 / \mathrm{s})$

Coefficient of volumetric thermal expansion of air $\left(1 /{ }^{\circ} \mathrm{K}\right)$

Dynamic viscosity (Pa.s)

Density $(\mathrm{kg} / \mathrm{m} 3)$

Fringe shift

Angle from stagnation point (degree)

Laser wave length $(\mathrm{m})$

Kinematic viscosity $(\mathrm{m} 2 / \mathrm{s})$

Refers to ambient condition

Refers to film condition

Refers to local value of parameter

Refers to reference condition

Refers to the cylinder surface 